\title{
Context effects in lexical access: A meta-analysis
}

\author{
MARGERY LUCAS \\ Wellesley College, Wellesley, Massachusetts
}

\begin{abstract}
The modularity hypothesis was evaluated in this review of 25 studies that investigated context effects in early lexical processing. Selection criteria restricted the review to priming studies and to studies that presented the target word before or at the end of the prime word. Meta-analysis was used to provide summary information about the 17 studies in the review for which effect sizes could be calculated. Overall, the analysis revealed a small effect of context on lexical access. Results provide a disconfirmation of the modularity hypothesis; although multiple interpretations of a word were sometimes accessed, the contextually appropriate interpretation was consistently more strongly activated. The lack of significant heterogeneity of variance suggests that the variability observed across studies was due to sampling error; however, task, timing of target presentation, meaning frequency, type of target, and type of context were identified as potential moderator variables.
\end{abstract}

Some of the most intense debates in psycholinguistics over the last 20 years have been about the architecture of the language-processing system. Although psycholinguists agree that there are a limited number of subsystems (the phonological/orthographic, lexical, syntactic, and conceptual) that contribute to language processing, there has been substantial argument about the relationships among these systems. On one view, the modularity hy pothesis, these subsystems are functionally autonomous; in other words, they are unaffected by feedback (Fodor, 1983; Forster, 1979). According to the alternative, the interactive hypothesis, feedback from higher levels of processing can influence the operation of lower subsystems (Marslen-Wilson \& Tyler, 1980; Marslen-Wilson \& Welsh, 1978).

The modularity hypothesis has been extensively investigated in studies of the phonological, lexical, and syntactic subsystems. The focus of this paper is on the lexical system. A number of techniques have been used to investigate modularity in lexical access, including priming, phoneme monitoring, and eye tracking. This review is concerned only with the priming studies. Focusing on just the priming studies avoids the interpretive difficulties inherent in comparing results across very different paradigms. Also, other techniques cannot discriminate among potential loci of processing load difficulties (Carpenter \& Daneman, 1981). Most models of lexical processing assume that there are three stages in that process: access

I am very grateful to David Pillemer, who first gave me the idea of doing a meta-analysis and who provided encouragement, advice, and support throughout the writing of this paper. I also thank Andrea Levitt for reading and commenting on an earlier draft of this paper. Greg Simpson and Mark St. John provided a number of suggestions that greatly improved this paper. Judy Hall and Jennifer Richeson kindly answered questions about meta-analytic procedures. However, I am the only one responsible for any errors or misunderstandings. Correspondence concerning this article should be addressed to M. Lucas, Department of Psychology, Wellesley College, Wellesley, MA 02481 (e-mail: mlucas@ wellesley.edu) of the lexical item and related information about the word, selection of the appropriate lexical item and related information given the context, and integration of that information with the sentential context. Evaluating the modularity hypothesis crucially depends on isolating the access component of lexical processing, rather than the selection or the integration component.

Priming tasks take advantage of the connection between related words in the lexicon: Participants are faster to respond to a word if it is preceded by a related word than if it is not. This fact is exploited in studies of lexical processing in which the target word follows another word (the prime) that is embedded in a sentence context. If access to the lexical representation of the prime is a modular process, targets related to all meanings of the prime word should be facilitated, relative to a control target. This is also known as the multiple access hypothesis. On the other hand, if lexical access is the result of interactive processes, only the target related to the contextually biased meaning should be facilitated (the selective access hypothesis). Overall, the results from studies that use priming to look for multiple or selective access are mixed. A number of studies indicate that lexical access is independent of context; others do not. Resolution of this issue has eluded the field owing to controversies over aspects of methodology, including task, timing, and type of context.

Most approaches to this issue focus on whether there is multiple or selective access. However, context effects are also reflected in the relative degree of activation of both the appropriate and the inappropriate targets. Simpson (1984), in a review of the literature, was the first to notice that there was a trend across studies for the appropriate target to be more primed than the inappropriate target, although this difference invariably failed to reach significance in any individual study. One reason for this failure may be that the test for multiple versus selective access is typically a main effect in which lexically related targets are compared with unrelated control targets, collapsing over appropriateness of targets in context. In contrast, a test of 
the size of the difference in degree of activation between appropriate and inappropriate targets is typically an interaction (involving appropriateness of target and relatedness), which has less power than the main effect to detect differences among conditions.

The existence of such an appropriateness effect would complicate the picture painted by advocates of multiple or selective access. The emphasis on multiple versus selective access in the literature has focused attention on extreme possibilities. On the one hand, the multiple access hypothesis supports modularity, in which lexical access is completely independent of context. Alternatively, the selective access hypothesis supports a strong interactive hypothesis, in which sufficiently biasing contexts can prevent access to an inappropriate interpretation. There is, however, a third possibility - a weaker form of interaction in which both appropriate and inappropriate interpretations are accessed but the appropriate interpretations receive greater priming (variations on this idea have been proposed by Simpson, 1984; Simpson \& Kreuger, 1991; Tanenhaus, Dell, \& Carlson, 1987). This last possibility may have received little verification in the literature because it involves small effects that are difficult to detect by conventional significance testing. But even a small effect of context is all that is needed to defeat modularity. The size of the appropriateness effect may vary, depending on the power of an individual study to detect the effect; if it is too small to reach significance in the test for the interaction, only multiple access would be detected. Small effects could also be responsible for reports of selective access. A strong context effect, such as that found in studies reporting selective access, may lead to very little priming for inappropriate targets. Although accessed, the inappropriate interpretation may not show significant priming if the power of a study to detect the priming effect is low.

The approach taken in the present article will be to first determine whether there is a consistent appropriateness effect across studies. This is accomplished using metaanalysis, a quantitative and comprehensive review of published and unpublished studies in which effects of interest are converted to standardized effect sizes (Glass, McGaw, \& Smith, 1981; Hedges \& Olkin, 1985; Light \& Pillemer, 1984; Rosenthal, 1984). The review is focused on studies that use priming techniques to determine the degree of activation of targets related to biased and unbiased readings of a word. In the review, the effect size (ES) for appropriate targets will be compared with the ES for inappropriate targets, to determine the degree of an appropriateness effect in each study. Even a small appropriateness effect, consistent across studies, would disconfirm modularity. The distribution of the standardized ESs will also be evaluated for indications that they arise from either a single population or different underlying populations of studies. Different populations of studies, based on variations in design or stimuli, might be responsible for greater context effects under certain conditions.

\section{METHOD}

\section{Selection of Studies}

In most of the studies chosen, lexical ambiguity was investigated. However, because the modularity and interactive hypotheses are not limited to ambiguity but are broadly concerned with context effects in lexical access (Swinney, 1979), studies that looked for activation of features associated with unambiguous words were also included.

The First Search and Psych Lit databases were canvassed for references, as were the literature review and discussion sections of published papers. Criteria for inclusion in the review included the following.

1. Only priming studies were included. Studies measuring processing load (phoneme monitoring, ambiguity detection) and eye movements were excluded for the reasons cited in the introduction. Priming tasks included lexical decision, naming, or color-naming (Stroop) tasks.

In a lexical decision task, participants hear or read a string of letters and indicate by a buttonpress whether or not the string is a word. Participants are faster to make a lexical decision if the string is preceded by a related word than if it is not. In a naming task, participants are asked to say the target word out loud. Latency to initiate this naming response is the measure of priming. In a color-naming task, targets are printed in different colors, and participants must name the color of the target, ignoring the word itself. Interference from the target word in naming the color is the measure of priming. Studies using the pseudoword priming task (Gildea, 1983; Glucksberg, Kreuz, \& Rho, 1986) were excluded because of questions about the sensitivity of that task to lexical priming (Burgess, Tanenhaus, \& Seidenberg, 1989). Likewise, Schustack's (1981) Experiments 1-4 were excluded because she used a word completion task that produced very long latencies, which suggests that the processing load in that task was disruptive to normal sentence processing (see also her discussion on p. 44 for other problems with that technique).

2. Experiments that used an interstimulus interval (ISI) longer than $0 \mathrm{msec}$ were excluded, to ensure that priming effects were measured at points most likely to reflect lexical access rather than later selection or integration processes.

3. Only studies using single prime words were included. Lexical access involving word combinations such as idioms were excluded because word combinations greatly amplify the problem of determining when initial access takes place.

\section{Procedure}

The search procedures outlined above located 25 references: 17 journal articles, 4 doctoral dissertations, 1 master's thesis, 2 book chapters, and 1 conference presentation. ${ }^{1}$ Most references included multiple experiments and/or multiple observations within an experiment. For example, noun-verb and noun-noun ambiguities might have been manipulated within a single experiment. Each manipulation was counted as a separate observation in the review, so that the study just described contained two observations, each with its own appropriateness effect.

Seventeen of the studies included information about variability from which to calculate an ES. There were a total of 52 observations across the 17 studies. However, not all observations were independent; some studies included many observations, and other studies only one. The problem of nonindependence can be avoided if all observations from a single study are averaged, so that each study has equal weight (Rosenthal, 1995). The individual observations are valuable, however. So ESs based on both individual observations and studies (combining observations) are reported.

ESs were calculated for the amount of priming for related targets in both appropriate and inappropriate contexts. Most studies reported priming for a target related to the ambiguous or unambiguous prime word, relative to a control item. Usually this control item 
was an unrelated target word matched in length and frequency to the related target word, but sometimes the latency to a target presented after a related prime was compared with the latency to the same target presented after an unrelated (control) prime. The targets were related either to the biased interpretation of the prime or to the unbiased interpretation of the prime. ESs were calculated both for priming for targets related to the biased interpretation (the appropriate target ) and for priming for targets related to the unbiased in terpretation (the inappropriate target). The ES for the inappropriate target was then subtracted from the ES for the appropriate target to get the appropriateness ES. A positive appropriateness ES indicated that the appropriate target showed relatively greater priming than the inappropriate target, a negative appropriateness ES that the inappropriate target showed greater priming than the appropriate target. For color naming, however, priming is indicated by inhibition rather than facilitation of the related target, so that the relationship between ESs for the appropriate and inappropriate target is reversed. For ease of comparison with other studies, the sign of the appropriateness ES for color-naming results was reversed, so that a positive appropriateness ES could be interpreted as greater faciiitation for the appropriate target. In a few studies, the researchers compared the amount of priming for the appropriate target with the amount of priming for the inappropriate target (without comparing each with a control item), to yield a more direct measure of the effect of target appropriateness. In these cases, the appropriateness ES was calculated with the means from this more direct comparison. The studies in this sample, along with their average ESs and characteristics, are listed in Appendix A. ${ }^{2}$

ESs were calculated as follows. Each observation was converted to a standard ES. When a study reported means and standard deviations, Cohen's $d$ was calculated, using Cohen's formula for the estimate of the pooled standard deviation (Cohen, 1977). When means and standard deviations were not provided, the square root of the $M S_{\mathrm{e}}$ of the main effect of relatedness (the comparison of related targets vs. control targets) was used to estimate the pooled standard deviation (Glass et al., 1981; Rosenthal \& Rosnow, 1991). If an exact $t$ for a planned comparison was reported, $d$ was estimated from $t$, using Rosenthal's conversion formula (Rosenthal, 1984; Rosenthal \& Rosnow, 1991). If a planned comparison with an exact $F$ was the only measure available, it was converted to a $t$ by taking the square root, and $d$ was estimated from the $t$, as was just described. ESs were calculated from results of subjects analyses only. In addition to ESs for individual observations, an overall mean ES was calculated for each study by taking the mean of all individual ESs in a study.

Nine studies that otherwise met the criteria for inclusion in the analysis did not provide any measure of variability from which to calculate an ES. ${ }^{3}$ These studies were not included in the metaanalysis proper; instead, they were included in vote counts of the direction of effects. These studies and their characteristics are listed in Appendix B. However, lacking an estimate of the magnitude of effect, these studies have a limited utility in the present analysis; the findings of the meta-analysis will draw primarily on the studies in the group providing ESs.

\section{RESULTS}

\section{Distribution of Effect Sizes}

ESs for the studies are provided in Figure 1. A striking observation about this display concerns the number of values on the positive side of 0 (no effect). According to the modularity hypothesis, if context has no effect on lexical access and error variance causes greater (numerical) priming in some appropriateness conditions than in others solely by chance, roughly half the observations should fall on the positive side (greater priming for appropriate targets) and half on the negative (greater priming for inappropriate targets). Yet, all but 1 of the 17 studies are on the positive side; the proportion of ESs on the positive side for the observations is $44 / 52$. These proportions were all significantly different from chance by chi-square goodness-of-fit tests. There were significantly more studies on the positive side $\left[\chi^{2}(1, N=17)=13.24, p<.001\right]$ and significantly more observations on the positive side $\left[\chi^{2}(1, N=52)=24.92, p<.001\right]$.

The studies with and without estimated ESs were combined to get overall proportions for the entire group. For the observations without ESs, an observation was counted as showing an appropriateness effect if there was numerically greater priming in the appropriate condition than in the inappropriate condition. Studies were counted as showing an appropriateness effect as long as the majority of observations in the study indicated that priming was greater for appropriate targets. The overall proportions are robustly in the positive direction: $59 / 76$ observations showed an appropriateness effect, and $21 / 26$ of the individual studies did as well. In other words, about $3 / 4$ of all observations and $4 / 5$ of all studies showed an appropriateness effect of some kind. These proportions were all significantly greater than expected by chance $\left[\chi^{2}(1, N=76)=\right.$ $23.21, p<.001$ by observations, and $\chi^{2}(1, N=26)=9.85$, $p<.01$ by studies].

\section{Central Tendency and Variability}

The median number of participants per observation was 32 , and the total number of participants across all observations was 2,106 . For the 52 observations, the mean ES was .20, and the median was .16. The mean ES for the 17 studies was .22 , and the median was .17. The weighted means were also calculated, using the sample size as a weighting factor. The weighted mean for both the observations and the studies was .18. There was little difference between weighted and unweighted means, so in the interest of parsimony, subsequent analyses use only the unweighted mean ES.

Fifty percent of the observations in the midrange had ESs between .07 and .40 (25th and 75 th percentile values). The range was narrower for the studies - between .07 and .29. The standard deviation for the unweighted mean of the observations was .27; for the studies, it was .25.

\section{Heterogeneity of Variance}

Although ESs were consistently positive, there is quite a bit of variability across studies. A critical question concerns whether this variability represents random sampling variation around a single, normally distributed ES value of roughly .2 or reflects the existence of two (or more) underlying populations that differ on some background factor or factors. These possibilities were investigated, using two tests for heterogeneity. In such tests, it is essential that ESs be independent. Therefore, because many observations were repeated measures, only the ESs from the study sample were used. 
Stem

1.0

Leaf

.9

.8

.7

.6

.5

$$
.4
$$

.3

$$
.2
$$

.1

.0

-0 .

-1 .
7

2

\section{8}

2

3

5

8

\section{9}

7

8

Note: This first column (the "stem") represents the first digit (or digits) of an effect size; the last digit is recorded in a "leaf" column to the right. To illustrate, the first three effect sizes (reading from the top) are 1.07, .42, and .48.

Figure 1. Stem and leaf display of effect sizes for 17 studies.

A graphic method for assessing heterogeneity of variance is provided by a funnel display (Light \& Pillemer, 1984). If studies are all from the same underlying population, then, as sample size increases, sampling error should decrease, and results should more accurately reflect a single underlying value. In Figure 2, ES is plotted against sample size. Although the sample of studies is small, the display does bears a striking resemblance to a funnel: There is much greater variation among ESs with small sample sizes (of 40 or less) than there is with larger sample sizes. The larger sample sizes converge on a small ES value in the range of .1-.2. This display suggests that the ESs in this sample are from a single population, with greater variation in the studies with smaller sample sizes; there is no suggestion of a nonnormal distribution.

A more formal test for heterogeneity of variance is the test statistic provided by Rosenthal and Rubin (1982). This test statistic is approximately distributed as a chi-square with $K-1 d f$, where $K$ is the number of ESs. Rosenthal and Rubin's formula applied to the study ESs yielded an approximate $\chi^{2}$ of 14.5 with $16 d f$ and is not significant. The results of both the funnel display and the heterogeneity of variance statistic, then, indicate that the ESs arise from a single underlying population value. This suggests that the variation across studies is simply due to sampling error. This is a surprising result, given the strong claims made in the literature that certain variables, such as frequency or strongly biasing contexts, can lead to significant differences in access to certain interpretations of a word.

However, a failure to find heterogeneity is no reason to refrain from looking for moderator variables (Light \& Pillemer, 1984; Rosenthal, 1995). Light and Pillemer argue that accepting the absence of heterogeneity has all the pitfalls of accepting the null hypothesis. In this case, the tests for heterogeneity may not have been powerful enough to detect variation, owing to the small sample size of 17 studies. Therefore, a search for moderator variables 
$100+$

90

80

70

Sample $\quad 60$

Size $\quad 50$

40

30

20

10 $\mathrm{x}$

$x$

$\mathbf{X}$

$\mathbf{x x}$

$\mathbf{x}$

$\mathbf{X}$

$\mathbf{x}$

$\mathbf{x x x x}$

$\mathbf{x}$

$\mathbf{x}$

$-.10$

.2

.3

.4

$.5+$

\section{Effect Size}

Figure 2. Funnel distribution of the appropriateness effect sizes for 17 studies.

seems warranted. This search is strictly exploratory but could provide clues to the most promising directions for future study.

\section{MODERATOR VARIABLES}

To assess the influence of moderator variables on context effects, the ESs were subdivided or blocked. Comparisons of blocked ESs should indicate whether samples differed in ES on the basis of differences in methodology or stimuli. Mean ESs were computed for each block both by observation and by study; the study ESs are reported in Table 1.

\section{Modality of Sentence Presentation}

Although there is no theoretical reason to suppose that context effects on lexical access might differ if sentences are heard rather than read, the modality of presentation of the sentence and the prime is a notable difference among the studies and may have affected the results. Some researchers used a cross-modal priming paradigm in which the sentence was heard and the target was presented visually; others preferred an all-visual presentation. But blocking on this variable indicated that modality was not responsible for variation in the studies. The ES across ob- servations for sentences presented visually $(.20)$ was almost identical to the ES for sentences presented auditorily (.21). Across studies, the ESs were slightly more disparate ( $\mathrm{ES}=.21$ for the sentences that were heard, as compared with .27 for the sentences that were read), but this disparity is no doubt due to skewing from the very small number of studies $(N=4)$ using the visual modality.

\section{Task}

Priming studies of lexical access typically make use of one of three priming tasks: lexical decision, naming, or color naming (also known as the Stroop task). Two of these tasks are controversial. Results from lexical decision tasks are problematic because they may be due to postlexical processes (Seidenberg, Waters, Sanders, \& Langer, 1984). Because lexical decision requires an explicit decision, responses may be contaminated by strategies that attempt to integrate the target with the context (a postaccess congruency check) in order to facilitate the decision. Also, lexical decision is sensitive to backward priming (Kiger \& Glass, 1983; Koriat, 1981). In the ideal priming experiment, the prime word will activate associated words, including the subsequent target if it is related (forward priming). However, the target may also trigger a backward association to the prime, in turn causing brief 
Table 1

Effect Sizes (ESs) and Characteristics of Experiments (By Studies)

\begin{tabular}{|c|c|c|c|}
\hline Characteristic & Average ES & $S D$ & $N$ of Studies \\
\hline \multicolumn{4}{|l|}{ Modality } \\
\hline All visual & .27 & .23 & 4 \\
\hline Cross-modal & .21 & .28 & 13 \\
\hline \multicolumn{4}{|l|}{ Task* } \\
\hline Lexical Decision & .23 & .30 & 10 \\
\hline Naming & .30 & .19 & 4 \\
\hline Color naming & .09 & .01 & 4 \\
\hline \multicolumn{4}{|l|}{ Length of context* } \\
\hline Short $(<6$ words $)$ & .22 & .13 & 4 \\
\hline Medium (6-10 words) & .33 & .38 & 6 \\
\hline Long (>10 words) & .15 & .09 & 6 \\
\hline \multicolumn{4}{|l|}{ Presentation Point* } \\
\hline End of word & .23 & .28 & 15 \\
\hline Prior to end of word & .11 & .10 & 5 \\
\hline \multicolumn{4}{|l|}{ Reaction Time } \\
\hline Short $(<600 \mathrm{msec})$ & .15 & .20 & 5 \\
\hline Medium (600-800 msec) & .30 & .31 & 8 \\
\hline Long $(>800 \mathrm{msec})$ & .12 & .03 & 3 \\
\hline \multicolumn{4}{|l|}{ Frequency } \\
\hline Dominant targets in inappropriate contexts & .22 & .20 & 5 \\
\hline Subordinate targets in inappropriate contexts & .21 & .24 & 5 \\
\hline Dominant targets overall & .28 & .10 & 5 \\
\hline Subordinate targets overall & .36 & .16 & 5 \\
\hline \multicolumn{4}{|l|}{ Type of Target } \\
\hline Associates & .16 & .19 & 7 \\
\hline Production features & .39 & .36 & 5 \\
\hline \multicolumn{4}{|l|}{ ContextType* } \\
\hline Constraining & .38 & .31 & 7 \\
\hline Undifferentiated & .13 & .15 & 10 \\
\hline Syntactic & .22 & & 1 \\
\hline
\end{tabular}

Note-An asterisk $\left(^{*}\right)$ indicates a condition in which the studies sum to greater than 17 because 1 study included observations that fall into more than one category of the characteristic.

facilitation for the target (backward priming). This latter facilitation is the result of the target-activating senses of the prime that would otherwise not be activated in normal sentence comprehension. Because lexical decision is more sensitive than the other tasks to postlexical processes and backward priming, its use introduces some uncertainty into the interpretation of results.

Seidenberg et al. (1984) demonstrated that, although lexical decision is sensitive to backward priming, naming is not. But naming does not always provide a pure measure of lexical access, either. Keefe and Neely (1990) found that naming is affected by expectancy, or the subject's ability to guess what the target might be. The colornaming task is less problematic than either lexical decision or naming, because it does not require a yes-or-no decision and has not yet been shown to be sensitive to either backward priming or expectancy effects. Given these concerns about some of the tasks, an examination of differences in ESs seemed warranted.

In both the observation and the study comparisons, the color-naming task showed a markedly smaller appropriateness effect than did either lexical decision or naming: The ES for the color-naming task was about half that for lexical decision and two thirds less than that for naming. This may be because the Stroop task is relatively insensitive to backward priming and to postlexical processes, which are somehow inflating the appropriateness ESs in the other tasks.

Greater vulnerability to backward priming may have contributed to the greater variability across studies in the lexical decision task. The standard deviation for that task was twice as large as that found in studies using either naming or Stroop tasks. Also, in the group of observations without ESs, almost all the naming observations showed an appropriateness effect $(9 / 10)$; only a little less than half of the lexical decision observations showed an appropriateness effect $(6 / 14)$.

The naming results, however, may have been contaminated by expectancy effects. Huttenlocher and Kubicek (1983) and Keefe and Neely (1990) found expectancy effects if the proportion of related to unrelated targets was quite high (88\%) but not if the proportion was low (33\%). Of the four studies that used naming, only one study (Jones, 1989a, Experiment 2) had a prime-target relatedness proportion of less than $33 \%$; this study also had the lowest appropriateness ES (ES $=.07)$ of any of the four naming studies. The other three studies, with higher ESs, had much higher proportions of prime-target relatedness: $50 \%$ for both Tanenhaus, Leiman, and Seidenberg (1979) and Simpson and Kreuger (1991). In Paul, Kellas, Martin, and Clark (1992), all prime-target pairs bore some relationship to each other. 
But, whereas strategic, postlexical processes might produce spurious lexical context effects, it is unclear how these would cause greater priming in the appropriate condition than in the inappropriate condition. Differences in the size of the appropriateness effect across tasks would have to be the result of more than simple expectations for certain targets generated by primes (for naming) or a cursory congruency check between prime and target (for lexical decision), because both appropriate and inappropriate targets are anticipated by or congruent with their ambiguous prime. It is only by considering the entire sentence context that one target would be favored over another. It is uncertain to what extent such comprehensive postlexical strategies were used by participants, given that the task set for them emphasized speed as well as accuracy. Nonetheless, it is possible that postlexical processes, in some experiments, boosted the appropriateness ESs for the lexical decision and naming tasks over that found in color naming.

\section{Length of Context}

The length of the sentences that preceded the prime varied considerably across studies. Some studies had very short sentences (fewer than 6 words); others had sentences with more than 10 words. If context effects take time to build, they may not show up in short contexts, as compared with longer ones (Tanenhaus \& DonnenwerthNolan, 1984). Tanenhaus and Donnenwerth-Nolan investigated the impact of context length by inserting a pause mid-sentence. They found multiple access in both the longer sentences and the shorter ones and concluded that participants did not need additional time to integrate the context. However, there are two reasons to suppose that the Tanenhaus and Donnenwerth-Nolan manipulation was an incomplete test of the hypothesis that context effects need more processing time than is available in short contexts. First, the difference between long and short sentences was just $400 \mathrm{msec}$, and this may not have been enough time to show the effects of context. Second, context effects might require a greater number of words before an effect is evident, not simply a greater amount of time.

The context-length hypothesis was tested in this review by comparing the ESs across three different context lengths: short (fewer than 6 words), medium (between 6 and 10 words), and long (more than 10 words). The context length for a study was the average of the number of words preceding (and not including) the prime in all the experimental sentences. Two studies, Swinney (1979) and Jones (1989b), did not report sentence length or provide a list of stimuli, so they were excluded from these comparisons.

The results show that longer contexts did not lead to larger ESs. The ES for long contexts (by observations) was .13; for short contexts, the ES was .21. By studies, the ES for long contexts was .15, as compared with .22 for the short contexts. The medium-length contexts had the largest ESs: $\mathrm{ES}=.27$ by observations, and $\mathrm{ES}=.33$ by studies. There is, thus, no evidence for the hypothesis that longer contexts increase the size of the context effect.

\section{Timing of Target Presentation}

As was noted in the introduction, an evaluation disconfirming the modularity hypothesis depends on tapping into the access component of lexical processing, rather than into the selection or integration component. The majority of the experiments in this review attempted to confine their results to the lexical access stage by presenting the target at the end of the prime. However, some studies looked at earlier presentation points, on the theory that access might be occurring earlier. If enough information was available prior to the end of the word to uniquely determine its identity, researchers using a 0 msec ISI may have been unintentionally tapping into the selection phase of lexical processing, rather than the access phase. Additional concerns about timing of the target presentation are raised by Simpson and Kreuger (1991), who note that sentence processing may be demanding enough to impede the participant's ability to shift quickly from the task of hearing or reading the sentence to one of making a response to a target. This could make a 0 -msec ISI functionally longer than the experimenter intended and place the participant's response in a postaccess stage. For these reasons, the ESs of studies using an early presentation point were compared with the ESs of studies using a standard 0 -msec ISI.

A common method for choosing the location of the target, relative to the prime, was simply to present targets at some fixed number of milliseconds after the onset of the prime. For example, Till et al. (1988) presented targets $200 \mathrm{msec}$ after prime onset $(200-\mathrm{msec}$ stimulus onset asynchrony $[\mathrm{SOA}])$. This method is less accurate than the gating paradigm in locating the access point. In the gating paradigm, the unique recognition point for each word is identified. Only one study (Moss and MarslenWilson, 1993) carefully controlled for the point of access by using a gating paradigm. However, for the purposes of the following comparisons, any SOA that presented the target prior to the end of the word was classified as an early presentation point.

Comparisons indicated that studies that used target presentation points closer to the access point showed less of an appropriateness effect: The ES for both observations and studies in which the target was presented before the end of the word was half as large as that for studies presenting targets at the acoustic offset of the word. The ES for the early condition was .11, as compared with .23 for the later condition (by study; the ESs by observations were almost identical to these values). This suggests that studies using later presentation points were actually tapping into the selection stage of lexical processing; if so, perhaps some of the studies that reported selective access were actually seeing the results of postaccess processes. This conclusion is tentative, however, because only one study used the gating paradigm to accurately locate the end 
of the word. It is possible, then, that some of the presentation points were too early for the access stage for some words. More studies that use the gating paradigm are needed, to confirm that there is a smaller appropriateness effect at the "true" point of access.

\section{Reaction Time}

If participants take a long time to respond, there is a danger that their responses will reflect postaccess rather than access processes (Picoult \& Johnson, 1992; Simpson \& Kreuger, 1991). To investigate this possibility, studies were blocked into three ranges of average response length: short (fewer than $600 \mathrm{msec}$ ), medium (between 600 and $800 \mathrm{msec}$ ), and long (greater than $800 \mathrm{msec}$ ). Reaction times (RTs) for both experimental and control targets were included in the average. One study did not report RTs (Jones, 1989a), so it was dropped from this analysis.

Longer RTs did not lead to greater context effects than either short or medium RTs. The ES for long RTs was essentially equivalent to the ES for short RTs, both in the comparison by observations ( $\mathrm{ES}=.15$ for long RTs, and $\mathrm{ES}=.13$ for short $\mathrm{RTs}$ ) and by studies (ES $=.12$ for long RTs, and ES $=.15$ for short RTs). In both comparisons, the ES for the medium-length RTs was the greatest at .27 by observations and at .30 by studies. This conclusion is supported by an examination of the studies without ESs. Of the observations in that group that report RTs, long RTs were actually less likely to show greater priming for the appropriate targets than were short or medium-length RTs. Only two out of seven observations in the long condition showed an effect in the right direction, as compared with five out of six of the observations in the short condition and four out of six of the observations in the medium condition.

\section{Frequency}

Ambiguous words usually have one interpretation (the dominant meaning) that is more frequent than another (the subordinate meaning). According to the ordered search hypothesis of Hogaboam and Perfetti (1975), the most frequent meaning is always accessed, regardless of context. If that meaning is appropriate, search terminates; if it is not, subordinate meanings are checked. Evidence for ordered access requires evidence that the subordinate interpretation is not accessed in a context that biases toward the dominant meaning, although the dominant interpretation is accessed in a context that biases toward the subordinate meaning.

There are comparatively few studies in the review that investigated the ordered search hypothesis. Of the studies using ambiguous words, equibalanced ambiguities were preferred. Only 10 studies used ambiguities that had both a dominant and a subordinate interpretation, and fewer still are in the ES group. A vote count shows that the evidence is somewhat mixed but that the majority of studies failed to find ordered access: Only 2 out of the 10 studies found evidence for ordered access. There were only 11 observations that allowed an ES to be computed for the dominant and subordinate interpretations in inappropriate contexts. The ES for subordinate targets in inappropriate contexts $(E S=.19)$ was very close to that for dominant targets in inappropriate contexts $(\mathrm{ES}=.23$ ). In the study comparison, the ES for targets related to the dominant meaning in the inappropriate context $(\mathrm{ES}=.22)$ is virtually identical to the ES for targets related to the subordinate meaning in the inappropriate context $(\mathrm{ES}=.21)$. These comparisons, then, provide no support for ordered access.

Simpson and Kreuger (1991) propose that, instead of order of access, frequency may influence speed of access. They found that the dominant meaning was accessed more quickly than the subordinate in ambiguous contexts (as did Li \& Yip, 1996). They suggested that speed of access is determined by degree of activation, which is, in turn, affected by both context and frequency (this view is similar to one expressed in Tanenhaus et al., 1987). On this view, the dominant meaning is accessed before the subordinate meaning because greater frequency leads to greater activation; but a strongly biasing context can raise the subordinate meaning's activation level to that of the dominant interpretation or even (in very strongly biasing contexts) cause its activation level to exceed the dominant's. This hypothesis could account for the failure of most studies to find frequency effects: When contexts lead to similar activation levels for both meanings, both will be accessed. If this hypothesis is correct, one would also expect that, on average, the dominant interpretation should show more priming than the subordinate interpretation. Because the dominant interpretation is more frequent, it should be activated to some extent in both dominant- and subordinatebiasing contexts. Subordinate interpretations, however, are less likely to be more activated than the dominant interpretation, unless the contexts have a strong bias toward those interpretations. To test this hypothesis, the ESs for the amount of priming (related target minus its control) for dominant and subordinate interpretations were compared. The priming ES for dominant targets across observations is essentially the same as the priming ES for subordinate targets (.33 and .32, respectively). The results of the study comparison are not as close, but the values are still similar at an ES of .28 for the dominant targets and . 36 for the subordinate targets (and in the reverse direction from what would be expected on Simpson \& Kreuger's frequency account).

Overall, these comparisons fail to support either an ordered access or a speeded access account of frequency effects in lexical access; however, this conclusion is tentative for two reasons. First, the comparisons involving ESs represent a small subset (seven) of the total number of studies. Second, only two studies (Paul et al., 1992; Simpson \& Kreuger, 1991) looked at frequency effects in ambiguous contexts. ${ }^{4}$ Simpson and Kreuger reported a substantial advantage of dominant targets over subordinate targets in nonbiasing contexts. They concluded that frequency information does affect lexical access but may be overwhelmed by a sufficiently strong context. However, Paul et al. failed to find differential priming for dom- 
inant over subordinate targets, perhaps because their ambiguous contexts were different from those of Simpson and Kreuger. Paul et al.'s ambiguous contexts were all semantically similar, in that each context was a variation on the theme that the upcoming target was a word (e.g. "You realize that the word is ..."). The small number of studies and the contradictory results of the two studies examining frequency effects in ambiguous contexts signal caution in drawing any definitive conclusions about frequency effects at this time.

\section{Type of Target}

The choice of target for a priming task may be critical to the size of the effects found. In studies that investigate single-word priming, targets that are associatively related to the prime are responsible for consistent and robust priming effects, whereas priming for semantically related targets is more variable (Hodgson, 1991; Lupker, 1984). If associates reflect cooccurrence relations in the lexicon that are responsible for fast and automatic access to related words (Fodor, 1983; Forster, 1979), it follows that they should be less susceptible to context effects than to other types of semantic relationships. Therefore, they should exhibit smaller appropriateness effects.

Although a number of studies in this review used associates for targets, others used targets generated by participants in a production task. In a production task, participants are asked to write down as many features as they can think of that are characteristic of a word. For example, in response to the word robin, participants might write bird and $f y$. These features are not necessarily associates, although they can be (and no studies reported that production features that were also associates were excluded). Associates and production features were the most common types of targets used in the studies. In some studies, the method for choosing targets was not well defined: Targets were described simply as "related" or "characteristic," but no systematic method of identifying these targets (outside of, presumably, the experimenter's intuition) was reported. Other studies mixed techniques and targets so that there was no single, readily identifiable class of targets in an experiment. These studies were excluded from the comparison of target types, which focused exclusively on studies using associates or production features.

As was expected, context affected associates less than it did production features. There was a much smaller appropriateness effect for associates (ES $=.13$ by observations and .16 by studies) than for production features ( $\mathrm{ES}=.28$ by observations and .39 by studies).

\section{Context}

All the studies in this review attempted to construct contexts that would bias toward one or the other interpretation of an ambiguous word or feature of an unambiguous word. But within this broad constraint, there was considerable variety in the types of context that were constructed. Several of the studies that reported significant context effects (i.e., selective access) attributed their find- ings to special features of the contexts used in their studies. The strongest claim is from Tabossi and her associates (Tabossi, 1988a, 1988b; Tabossi, Colombo, \& Job, 1987; Tabossi \& Zardon, 1993), who claim that selective access is most likely to occur in what Tabossi and Zardon called constraining contexts. These are contexts which bias toward an interpretation by "rendering salient one central aspect of that meaning" (Tabossi \& Zardon, 1993, p. 362). In comparison, Tabossi claims, the contexts in studies such as Whitney, McKay, Kellas, and Emerson (1985) or Onifer and Swinney (1981) were inadequate for inducing selective access because, although these contexts biased toward one interpretation of the prime, they imposed insufficient constraints on the semantic information that needed to be retrieved. Tabossi (1988b) gives as an example this sentence from Whitney et al.: "The man used the oak." In that context, "The man used the ..." is too vague to restrict access of information about oak to the target lumber. But Tabossi's (1988b) context "In the light, the blond hair of the little girl had the lustre of gold" should be sufficient to prime the target yellow selectively. The comparisons reported below evaluate claims about the power of constraining contexts, as opposed to unconstrained contexts, in limiting lexical access to appropriate interpretations.

Researchers who constructed constraining contexts all followed similar norming procedures to find features that were constrained by the context. In these experiments, the contexts were evaluated by judges (other than the experimenters), who were asked to suggest which features of the ambiguous or unambiguous words were emphasized by the context. Features that most judges thought were stressed by a particular context were chosen to be targets. All the studies that used this technique to generate biasing contexts were included in the constraining context category. ${ }^{5}$

The remaining studies reported no systematic criterion for constructing contexts, and many failed to gather normative data on the contexts. Some of these contexts were constructed by the experimenters, presumably using their own intuitions about what would count as biasing. Some asked participants for their judgments about whether a context was biasing but gave no instruction on what would count as biasing and did not have judges suggest targets that were constrained by the context. Most of these contexts biased through plausibility or predictability. Because contexts may be plausible or predictive in many different ways, this category represents quite a mixture of context types. For ease of reference, these contexts will simply be called undifferentiated.

Constraining contexts had a mean ES twice as large as that for undifferentiated contexts by observation (ES = .29 for constraining contexts and .12 for undifferentiated ones) and three times as large by study ( $\mathrm{ES}=.38$ for constraining contexts and 13 for undifferentiated ones). The group without ESs has a similar profile: Four out of $5 \mathrm{ob}-$ servations using constraining contexts show an effect in the right direction (greater priming for the appropriate targets), but only 5 out of 10 observations using undifferentiated contexts do. These comparisons strongly support 
the claims of researchers who argue that constraining contexts are more likely to produce context effects on access. Note, however, that such contexts are not necessary to produce context effects. Jones (1989a) and Li and Yip (1996) report selective access without constraining contexts, and even the undifferentiated group shows an average small ES of .13. Also, constraining contexts were not always sufficient to produce selective access: Neither Moss and Marslen-Wilson (1993) nor Swinney (1991) reports selective access for appropriate targets in their constraining contexts.

Only one study in the group of studies with ESs investigated the role of syntactic context on lexical access (Tanenhaus et al., 1979). Their contexts biased toward one interpretation of a noun-verb ambiguity by manipulating the expected word class of the prime. For example, "I bought the watch" biases toward the noun interpretation of watch, because the determiner the sets up an expectation that a noun (or an adjective), not a verb, will follow. The average ES for that study (with two observations) was .22, which matches the overall ES for the entire sample. Three studies in the group without ESs investigated syntactic contexts (Schustack, 1981; Seidenberg, Tanenhaus, Leiman, \& Bienkowski, 1982, Experiments 3 and 4; Tanenhaus \& Donnenwerth-Nolan, 1984). Out of seven observations across the two groups, four are in the right direction. It may be that syntactic contexts do give rise to a small context effect, but it is difficult to feel confident about this conclusion, given the extremely small number of observations.

\section{GENERAL DISCUSSION}

The priming studies in this review showed a small effect of context on lexical access of about two tenths of a standard deviation: The appropriate interpretation of a word consistently showed greater priming than the inappropriate interpretation. Even this small ES is sufficient to disconfirm the modularity hypothesis. Moreover, despite claims by various researchers that certain variables influence access, the results of heterogeneity of variance tests suggested that the wide variation in ESs across studies may have been due simply to sampling error.

This kind of variation is to be expected, given the low power in these studies to detect small effects. To detect an effect of .2 at just greater than chance probability $(.51)$ with an alpha of .05 , the $N$ of a study would have to be 140 (from Cohen's, 1977, power tables). None of the sample sizes in any of the studies reviewed was that high. The median sample size of an experiment was 35 , and the largest sample in any one experiment was 127 . This means that, in half of the experiments in this review, the power to detect a small ES of . 2 was, at best, just .13; in the experiment with the largest sample, the power to detect a small effect was just .38. One recommendation for future research, then, would be to increase the number of participants in an experiment, or at least to report ESs; this would facilitate comparisons with other studies, as compared with the cur- rent practice of only making a categorical decision about significance.

A search for moderator variables gave no indication that modality of sentence presentation, length of context, and length of response influenced access. There was no evidence for ordered access or faster access for dominant interpretations in biasing contexts. However, there were relatively few studies in the review that manipulated meaning frequency, and fewer still that included ambiguous contexts in their manipulations. This indicates a need for more attention to frequency in future research.

There were indications that type of task and presentation point might influence the probability of finding a context effect. The use of the Stroop task as a measure of priming reduced the size of the appropriateness effect, which may indicate that acknowledged problems with the naming and lexical decision tasks (expectancy effects, backward priming, and the like) are contaminating the results of at least some of the studies. However, later studies were more aware of and, hence, more vigilant against potential contaminating factors in these tasks. Moreover, color naming has been used less frequently and has not been as carefully scrutinized as either of the other tasks, so its potential flaws may not yet be apparent. ${ }^{6}$ It is also possible that it is less sensitive to appropriateness effects.

Presenting the target prior to the end of the word seemed to reduce the size of the appropriateness effect. This suggests that a 0 -msec ISI might be too late to capture the initial stages of lexical access. However, because most of the studies using earlier presentation points were somewhat haphazard in their selection of presentation point, more use of the gating paradigm is needed to resolve this issue.

Variations in target type seemed to be responsible for some of the variation in context effects in lexical access. Associates in our sample led to weaker context effects than did other types of targets, although they were still responsible for a weak appropriateness effect. This finding provides more evidence against modularity. According to Fodor (1983), the lexicon encodes only co-occurrence information among words; once a word is activated, linked lexical items should be obligatorily activated as well. An appropriateness effect for associates raises problems for this hypothesis.

Aside from the studies using associates, there were few semantic constraints on the type of targets chosen, so the targets were quite mixed in type. The loose restrictions on the type of targets used in many of the studies in this review may have contributed to the variation in the degree of context effects observed across studies. This lack of rigor, in turn, may reflect inadequate theories of word meaning; word meaning was ill defined in many of these papers. Targets were sometimes chosen solely by intuition but, more frequently, by just asking participants to generate features for words. More progress in the study of lexical access might be made by following the lead of Moss and Marslen-Wilson (1993), who used more principled distinctions among targets on the basis of core and periph- 
eral aspects of word meaning. Although the distinctions they drew were based on a theory of semantic representation (cognitive economy in semantic networks) that is problematic (Ashcraft, 1976; Collins \& Loftus, 1975), the attempt to move beyond intuition represents a step in the right direction.

The most dramatic claims for context effects has come from researchers who used constraining contexts, which are contexts that bias an interpretation by activating specific features of a noun's meaning. Although, as was discussed earlier, constraining contexts are not necessary to produce context effects (or even selective access), they do appear to be responsible for larger ESs than are undifferentiated contexts. The exceptions to this rule are the studies by Moss and Marslen-Wilson (1993) and Swinney (1991), which also used contexts that were designed to constrain the interpretation of the prime to one aspect of its meaning, but which failed to find significant effects of appropriateness. Swinney (1991) has suggested that this discrepancy is due partly to Tabossi's materials and partly to the fact that her stimuli were in Italian. However, other studies (Kellas, Paul, Martin, \& Simpson, 1991; Paul, 1992, and Paul et al., 1992) that report selective access with constraining contexts use stimuli that are both different from Tabossi's and in English. In light of these discrepancies in results across studies, an account of what makes one researcher's constraining contexts different from another's is needed.

An obvious first place to look is in the procedures used to generate constraining contexts. Generally, all the constraining contexts were first generated by the experimenter; then, judges were asked to confirm the experimenter's intuitions that each sentence restricted the interpretation of the prime word to some attribute of its meaning. A priori constraints on the specific nature of these contexts were minimal. Experimenters avoided sentences that contained a strongly associated word that might prime the target even in the absence of the prime, as well as contexts that were strongly predictive of the prime itself, as opposed to an attribute of it. Also, as Swinney (1991) notes, in Tabossi's studies, judges were simply asked to use their intuitions and were given no theoretical guidance regarding what makes a context constraining; this was true for most of the studies in this category. These very broad constraints led to quite a range of contexts. For example, some contexts (those in Moss \& Marslen-Wilson, 1993; Swinney, 1991; Tabossi, 1988a, 1988b; Tabossi et al., 1987; Tabossi \& Zardon, 1993) all seemed to make a particular feature salient through the use of weakly associated or related words. Alone, these words would not strongly suggest a particular feature, but together they made alternative features implausible. The example provided below illustrates this (the prime is the last word in the sentence and the target is the word in capital letters following it). Italicized words are words that are weakly associated or otherwise related to the target.
The violent hurricane did not damage the ships which were in the port ... SAFE (from Tabossi, 1988a)

Other contexts, such as those in Kellas et al. (1991), Paul (1992), and Paul et al. (1992), seemed to be much less restrictive (see example below).

The girl's bag was made of straw . . HAY. (from Kellas et al., 1991)

In the example above, there is nothing in the context "The girl's bag was made of ..." that makes hay as a feature of straw particularly salient. Of the studies that had the weakly associative type of context, only Tabossi's contexts showed selective access; all the researchers using the much less restrictive contexts found significant context effects. Comparing these two very different types of contexts, it would seem that the only feature in common among these various constraining contexts is that they are self-identified as constraining.

Swinney (1991) suggests that Tabossi (and, by implication, others who use constraining contexts and find selective access) may not have had the power in their studies to detect priming of the inappropriate target. This is a point worth considering. The ESs for priming effects (observations only) were a moderately sized ES of .37 for priming of appropriate targets but a much smaller ES of .20 for inappropriate targets. Given these ESs, it is much more likely that an experimenter will find significant effects of priming of the appropriate target, rather than of the inappropriate target. But, as has been argued in this review, it is also true that the studies lack sufficient power to find a significant appropriateness effect. The low power to detect priming for inappropriate targets (leading to a failure to detect multiple access) is accompanied by low power to detect an appropriateness effect (leading to a failure to detect greater priming for the appropriate target). So, if the variation across studies is due to sampling error, as the results of the heterogeneity of variance tests suggest, rather than to the manipulations of the experimenters, the fact that constraining contexts do not always show significant context effects and unconstraining contexts sometimes do is not so surprising. In fact, given the relatively small sample sizes in all of these studies, it is to be expected.

These conclusions do not rule out the possibility that differences in types of contexts might yet reveal consistent and reliably greater context effects in the form of selective access (no priming for the inappropriate target), as opposed to a weaker form of context effect in which there is multiple access with greater priming for appropriate interpretations. But findings like these await the development of more stringent criteria for defining contexts.

\section{REFERENCES}

AshCraft, M. H. (1976). Priming and property dominance effects in semantic memory. Memory \& Cognition, 4, 490-500.

Burgess, C., Tanenhaus, M. K., \& Seidenberg, M. S. (1989). Context and lexical access: Implications of nonword interference for lex- 
ical ambiguity resolution. Journal of Experimental Psychology: Learning, Memory, \& Cognition, 15, 620-632.

Carpenter, P. A., \& DANEMAN, M. (1981). Lexical retrieval and error recovery in reading: A model based on eye fixation. Journal of Verbal Learning \& Verbal Behavior, 20, 137-160.

COHEN, J. (1977). Statistical power analysis for the behavioral sciences. New York: Academic Press.

Collins, A. M., \& LofTus, E. J. (1975). A spreading activation theory of semantic processing. Psychological Review, 82, 407-428.

FODOR, J. A. (1983). The modularity of mind: An essay on faculty psychology. Cambridge, MA: MIT Press.

*ForemAN, J. G. (1992). Evidence for the multiple access model of lexical access using strong sentential context. Unpublished master's thesis, University of Alabama, Huntsville.

FORSTER, K. I. (1979). Levels of processing and the structure of the language processor. In W. E. Cooper \& E. C. T. Walker (Eds.), Sentence processing: Psycholinguistic studies presented to Merrill Garrett (pp. 257-287). Cambridge, MA: MIT Press.

*GILDEA, P. (1983). On resolving ambiguity: Can context constrain lexical access? Unpublished doctoral dissertation, Princeton University, Princeton, New Jersey.

Glass, G. V., McGaw, B., \& Smith, M. L. (1981). Meta-analysis in social research. Beverly Hills, CA: Sage.

Glucksberg, S., Kreuz, R. J., \& RHo, S. (1986). Context can constrain lexical access: Implications for models of language comprehension Journal of Experimental Psychology: Learning, Memory, \& Cognition, 12, 323-335.

HedGes, L. V., \& OLKIN, I. (1985). Statistical methods for meta-analysis. New York: Academic Press.

HoDGSON, J. (1991). Informational constraints on pre-lexical priming. Language \& Cognitive Processes, 6, 169-205.

Hogaboam, T. W., \& Perfetti, C. A. (1975). Lexical ambiguity and sentence comprehension. Journal of Verbal Learning \& Verbal Be havior, 14, 265-274.

HUtTENLOCHER, J., \& KUBICEK, L. (1983). The source of relatedness effects on naming latency. Journal of Experimental Psychology: Learning, Memory, \& Cognition, 9, 486-496.

*JoNES, J. L. (1989a). The interactive lexicon: Context-dependent access of homonym meanings. Unpublished doctoral dissertation, University of California, Los Angeles.

*JONES, J. L. (1989b). Multiple access of homonym meanings: An artifact of backward priming? Journal of Psycholinguistic Research, 18 417-432.

Keefe, D. E., \& NeELy, J. H. (1990). Semantic priming in the pronunciation task: The role of prospective prime-generated expectancies Memory \& Cognition, 18, 289-298.

*Kellas, G., Paul, S. T., Martin, M., \& Simpson, G. B. (1991). Contextual feature activation and meaning access. In G. B. Simpson (Ed.), Understanding word and sentence (pp. 47-71). New York: North-Holland.

Kiger, J. I., \& Glass, A. L. (1983). The facilitation of lexical decisions by a prime occurring after the target. Memory \& Cognition, 11, 356365 .

*Kintsch, W., \& Mross, E. (1985). Context effects in word identification. Journal of Memory \& Language, 24, 336-349.

Koriat, A. (1981). Semantic facilitation in lexical decision as a function of prime-target association. Memory \& Cognition, 9, 587-598.

*LI, P., \& YIP, M. C. (1996). Lexical ambiguity and context effects in spoken word recognition: Evidence from Chinese. In Proceedings of the Eighteenth Annual Conference of the Cognitive Science Society (pp. 228-232) Mahwah, NJ: Erlbaum

Light, R. J., \& PillemER, D. B. (1984). Summing up: The science of reviewing research. Cambridge, MA: Harvard University Press.

LUPKER, S. J. (1984). Semantic priming without association: A second look. Journal of Verbal Learning \& Verbal Behavior, 23, 709-733.

MARSLEN-Wilson, W., \& TYler, L. K. (1980). The temporal structure of spoken language understanding. Cognition, 8, 1-71.

Marslen-Wilson, W., \& Welsh, A. (1978). Processing interactions and lexical access during word recognition in continuous speech. Cognitive Psychology, 10, 29-63.

*Moss, H. E., \& MARSLEN-Wilson, W. D. (1993). Access to word mean- ings during spoken language comprehension: Effects of sentential semantic context. Journal of Experimental Psychology: Learning. Memory, \& Cognition, 19, 1254-1276.

*ONIFER, W., \& SwinNey, D. A. (1981). Accessing lexical ambiguities during sentence comprehension: Effects of frequency of meaning and contextual bias. Memory \& Cognition, 9, 225-236.

*Paul, S. T. (1992). Sentence priming effects on lexical access and postaccess processes. Unpublished doctoral dissertation, University of Kansas, Lawrence.

*Paul, S. T., Kellas, G., Martin, M., \& Clark, M. B. (1992). Influence of contextual features on the activation of ambiguous word meanings. Journal of Experimental Psychology: Learning, Memory, \& Cognition, 18, 703-717.

*Picoult, J., \& Johnson, M. K. (1992). Controlling for homophone polarity and prime-target relatedness in the cross-modal lexical decision task. Bulletin of the Psychonomic Society, 30, 15-18.

RoSENTHAL, R. (1984). Meta-analytic procedures for social research. Beverly Hills, CA: Sage.

Rosenthal, R. (1995). Writing meta-analytic reviews. Psychological Bulletin, 118, 183-192.

Rosenthal, R., \& RosNow, R. L. (1991). Essentials of behavioral research: Methods and data analysis. New York: McGraw-Hill.

Rosenthal, R., \& Rubin, D. B. (1982). Comparing effect sizes of independent studies. Psychological Bulletin, 92, 500-504.

*SchustaCK, M. W. (1981). Word-meaning comprehension: Syntactic and associative effects of sentential context. Unpublished doctoral dissertation, Carnegie-Mellon University, Pittsburgh.

*Seidenberg, M. S., Tanenhaus, M. K., Leiman, J. M., \& BienKowsKI, M. (1982). Automatic access of the meanings of ambiguous words in context: Some limitations of knowledge-based processing. Cognitive Psychology, 14, 489-537.

Seidenberg, M. S., Waters, G. S., Sanders., M., \& Langer, P. (1984). Pre- and postlexical loci of contextual effects on word recognition. Memory \& Cognition, 12, 315-328.

Simpson, G. B. (1984). Lexical ambiguity and its role in models of word recognition. Psychological Bulletin, 96, 316-340.

*SimpSon, G. B., \& KREUGER, M. A. (1991). Selective access of homograph meanings in sentence context. Journal of Memory \& Language, 30, 627-643.

*SWinNEY, D. A. (1979). Lexical access during sentence comprehension: (Re)Consideration of context effects. Journal of Verbal Learning \& Verbal Behavior, 18, 645-659.

*SwinNEY, D. A. (1991). The resolution of interdeterminacy during language comprehension: Perspectives on modularity in lexical, structural, and pragmatic processes. In G. B. Simpson (Ed.), Understanding word and sentence (pp. 357-384). New York: North-Holland.

*TaBOSSI, P. (1988a). Accessing lexical ambiguity in different types of sentential contexts. Journal of Memory \& Language, 27, 324-340.

*TaBossi, P. (1988b). Effects of context on the immediate interpretation of unambiguous words. Journal of Experimental Psychology: Learning, Memory, \& Cognition, 14, 153-162.

*TaBossi, P, Colombo, L., \& JoB, R. (1987). Accessing lexical ambiguity: Effects of context and dominance. Psychological Research, 49, 161-167.

*TABOssi, P., \& ZARDON, F. (1993). Processing ambiguous words in context. Journal of Memory \& Language, 32, 359-372.

Tanenhaus, M. K., Dell, G. S., \& Carlson, G. (1987). Context effects and lexical processing: A connectionist approach to modularity. In J. L. Garfield (Ed.), Modularity in knowledge representation and natural-language understanding. Cambridge, MA: MIT Press.

*Tanenhaus, M. K., \& Donnenwerth-Nolan, S. (1984). Syntactic context and lexical access. Quarterly Journal of Experimental Psychology, 36A, 649-661

*Tanenhaus, M. K., Leiman, J. M., \& Seidenderg, M. S. (1979). Evidence for multiple stages in the processing of ambiguous words in syntactic contexts. Journal of Verbal Learning \& Verbal Behavior, $18,427-440$

*Till, R. E., Mross, E. F. \& KinTsCh, W. (1988). Time course of priming for associate and inference words in a discourse context. Memory \& Cognition, 16, 283-298.

*Whitney, P., MCKay, T., Kellas, G., \& Emerson, W. A., JR. (1985). 
Semantic activation of noun concepts in context. Journal of Experimental Psychology: Learning, Memory, \& Cognition, 11, 126-135. *Indicates one of the priming studies included in this review.

\section{NOTES}

1. Several other conference presentations were located, but either they were based on doctoral dissertations already included in the review or they reported data later included in journal articles.

2. To conserve space, the information about individual observations is not included in the appendices or in any subsequent tables and figures. This information can be obtained by writing to the author.

3. This group included one study (Till, Mross, \& Kintsch, 1988) that was also included in the group that permitted ES calculations. Experiment 1 of that study did not report any measure of variability from which to calculate an ES, but Experiments 2 and 3 did. So these three experiments were split between the two samples accordingly
4. My thanks to Greg Simpson for bringing this condition to my attention.

5. This criterion excluded from this classification Whitney et al. (1985), whose description of the process of context construction indicates that he was attempting to construct constraining contexts. But he used only his own intuitions, rather than the intuitions of a group of native speakers. Because an experimenter's intuition is not always reliable, in the following comparisons, contexts were only categorized as constraining contexts if they had been evaluated by norms gathered from judges who were not also experimenters. This criterion also excludes Simpson and Kreuger (1991), who argue that their strongly biasing contexts probably would qualify as constraining, but who did not use the specific criteria for constraining contexts outlined here. Loosening these criteria to include these two studies in the constraining condition did not substantially change the results of the analysis; so, just the results from using the stricter criterion are reported here.

6. I am grateful to Mark St. John for pointing out this possibility to me. 

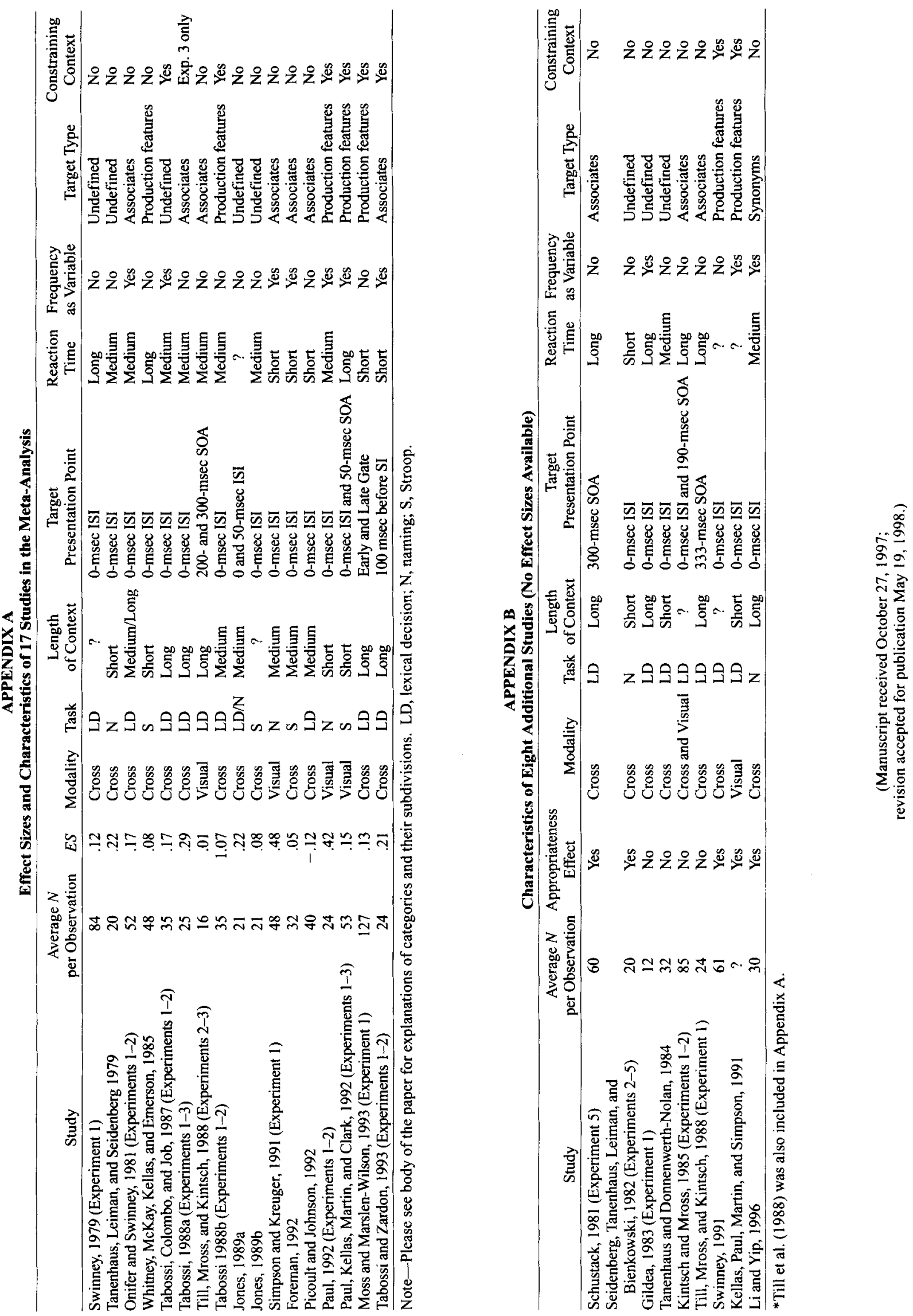